\title{
Comment on: “Cost-Effectiveness of Niraparib Versus Routine Surveillance, Olaparib and Rucaparib for the Maintenance Treatment of Patients with Ovarian Cancer in the United States"
}

\author{
Katrine Wallace $^{1} \cdot$ Sandra Goble ${ }^{1} \cdot$ Jeff Isaacson $^{1} \cdot$ Lara Maloney $^{1} \cdot$ Terri Cameron $^{2} \cdot$ Josh Bedel $^{3}$
}

Published online: 7 June 2019

(c) The Author(s) 2019

\section{Dear Editor,}

We read with interest the article by Guy et al. [1] detailing a cost-effectiveness model comparing niraparib, a poly(ADPribose) polymerase inhibitor (PARPi), to routine surveillance and two other PARPis approved for maintenance treatment of recurrent ovarian cancer, olaparib and rucaparib. The authors concluded from their analysis that niraparib was less expensive and more cost effective than olaparib and rucaparib, and the incremental cost-effectiveness ratio (ICER) fell within an acceptable range compared to routine surveillance. However, we noted several fundamental problems with the lack of comparability of populations, as well as methodological flaws and violations of statistical assumptions within the model. Although we found the authors' methods flawed overall, in this letter, we comment only on the main problems with the comparison between niraparib and rucaparib.

The authors mention that the analysis of niraparib vs. rucaparib is "a naïve side-by-side comparison" of results from the ENGOT-OV16/NOVA study for niraparib [2] and the ARIEL3 study for rucaparib [3]. A naïve indirect comparison or analysis of two treatment groups as if they were from a single trial without adjustment for potential betweentrial variability is not a recommended method of analysis because the homogeneity assumption is nearly impossible to meet [4]. For the homogeneity assumption to be met, the

This comment refers to the article available at https://doi. org/10.1007/s40273-018-0745-z.

Katrine Wallace

kwallace@clovisoncology.com

1 Clovis Oncology, Inc., 5500 Flatiron Pkwy, Boulder, CO 80301, USA

2 Clovis Oncology UK Ltd., Cambridge, UK

3 Clovis Oncology Switzerland GmBH, Zurich, Switzerland groups being compared should not have been selected with different inclusion/exclusion criteria or undergone data collection via different methodologies [4]. Throughout their paper, the authors violate this assumption, rendering the model and results invalid.

First, the niraparib and rucaparib populations analyzed within the cost-effectiveness model are dissimilar. Somatic $B R C A$-mutant (BRCAmut) patients were included in the "non-germline $B R C A$ (non-g $B R C A$ )" group for the niraparib data [2], but they were classified in the BRCAmut group for the rucaparib data [3]. This dissimilarity leads to bias in assessing efficacy because somatic BRCAmut patients have been shown to respond comparably to $\mathrm{g} B R C A$ mut patients (and better than wild-type $B R C A$ patients) in the maintenance and treatment settings of recurrent ovarian cancer [2, $3,5,6]$. For the non-gBRCA group, the median progression-free survival (mPFS) of 9.3 months based on a blinded independent central review (BICR) was used for niraparib, whereas a weighted average of the wild-type $B R C A / g e n o m i c$ high loss of heterozygosity and wild-type $B R C A /$ loss of heterozygosity low mPFS numbers ( 8.2 months) was used for rucaparib because ARIEL3 did not report a MPFS for non$\mathrm{g} B R C A$ patients at the time of the primary analysis. These data have been reported recently for rucaparib [7] and show higher mPFS for the non-gBRCA population (11.1 months for BICR-assessed mPFS and 8.6 months for investigatorassessed mPFS) than that calculated by Guy et al. [1].

Furthermore, the efficacy assessments used for the model are also not comparable. Their model included the BICRassessed mPFS for niraparib gBRCA (21.0 months) [2] vs. investigator-assessed mPFS (16.6 months) for rucaparib [3]. In three phase III studies investigating PARPis for maintenance treatment of recurrent ovarian cancer, MPFS was longer in BICR-assessed than investigator-assessed progression-free survival (PFS) [2, 3, 8]. Moreover, results from these three studies demonstrate that inconsistency in efficacy assessments used in the model by Guy et al. leads to bias in 
the overall results. Given that BICR-assessed PFS data have been reported for all three PARPis, those data should have been used consistently across all treatment groups in the analysis. Thus, the more appropriate comparator for rucaparib is the BICR-assessed PFS for patients with BRCAmut disease: 26.8 months. In the case of rucaparib, the BICRassessed mPFS is almost 10 months longer than investigatorassessed mPFS [3].

The adverse event (AE) data used in the treatment cost calculations were also not presented uniformly for each agent. For example, the authors only included the rates of grade 3-4 niraparib-associated thrombocytopenia (33.8\%) in their cost analysis [1]. In contrast, they present dose interruption or reduction data for any-grade (i.e., grades 1-5) rucaparib-associated thrombocytopenia (18.0\%). The correct comparator should have been the rate of grade 3-4 rucaparib-associated thrombocytopenia (5.1\%) [3]. Across the AE data, the authors presented the rates of dose interruption or reductions for any-grade AEs for rucaparib instead of the grade 3-4 AE rates they presented for niraparib.

We also identified methodologic issues and inappropriate assumptions within the model itself. For example, the authors assumed that overall survival duration is two times that of PFS, based on what was seen in a phase II study (Study 19) of olaparib in recurrent ovarian cancer [8]. The relationship between overall survival and PFS has not been demonstrated sufficiently in the literature. In fact, the National Institute for Health and Care Excellence negatively critiqued the use of the overall survival calculation from Study 19 in their own review of the authors' model [9].

Additionally, the cost/efficacy data in their ICER calculation are mismatched. The analysis presents PFS data for niraparib based on the starting dose of $300 \mathrm{mg}$ once daily; however, the cost data were calculated for the step-down dose of niraparib $200 \mathrm{mg}$ once daily (based on the dose reduction rate observed with niraparib). Therefore, the ICER calculation uses different data scenarios for niraparib in the numerator than that in the denominator:

ICER $=\frac{\Delta \text { Costs (based on step-down niraparib dose })}{\Delta \text { Efficacy (PFS based on starting niraparib dose) }}$.

Although their cost analysis was based on the rate of dose reductions for niraparib, the dose reduction rate for rucaparib was not similarly considered; thus, Guy et al. compare the starting dose for rucaparib against the modal dose for niraparib, which is not a valid comparison.

In summary, we observed several comparability problems that violate homogeneity assumptions, as well as inconsistent data presentation and previously documented methodological flaws within the Guy et al. model. All of our observations seriously call into question the validity of the conclusions the authors draw from their analysis, including whether niraparib is in fact the most cost-effective agent. It is essential when making comparisons between trials that proper statistical methods are employed, data are selected carefully and consistently, and proper adjustments are made to ensure the control of inter-trial variability.

Acknowledgements Copy editing assistance funded by Clovis Oncology, Inc., was provided by Nathan Yardley and Shannon Davis of Ashfield Healthcare Communications (Middletown, CT, USA).

\section{Compliance with Ethical Standards}

Funding Funding was provided by Clovis Oncology, Inc.

Conflict of interest Katrine Wallace, Sandra Goble, Jeff Isaacson, Lara Maloney, Terri Cameron, and Josh Bedel are employees of Clovis Oncology, Inc., and may own or have stock options in the company.

Open Access This article is distributed under the terms of the Creative Commons Attribution-NonCommercial 4.0 International License (http://creativecommons.org/licenses/by-nc/4.0/), which permits any noncommercial use, distribution, and reproduction in any medium, provided you give appropriate credit to the original author(s) and the source, provide a link to the Creative Commons license, and indicate if changes were made.

\section{References}

1. Guy H, Walder L, Fisher M. Cost-effectiveness of niraparib versus routine surveillance, olaparib and rucaparib for the maintenance treatment of patients with ovarian cancer in the United States. Pharmacoeconomics. 2019;37:391-405.

2. Mirza MR, Monk BJ, Herrstedt J, et al. Niraparib maintenance therapy in platinum-sensitive, recurrent ovarian cancer. N Engl J Med. 2016;375:2154-64.

3. Coleman RL, Oza AM, Lorusso D, et al. Rucaparib maintenance treatment for recurrent ovarian carcinoma after response to platinum therapy (ARIEL3): a randomised, double-blind, placebocontrolled, phase 3 trial. Lancet. 2017;390:1949-61.

4. Glenny AM, Altman DG, Song F, et al. Indirect comparisons of competing interventions. Health Technol Assess. 2005;9:1-134.

5. Swisher EM, Lin KK, Oza AM, et al. Rucaparib in relapsed, platinum-sensitive high-grade ovarian carcinoma (ARIEL2 Part 1): an international, multicentre, open-label, phase 2 trial. Lancet Oncol. 2017;18:75-87.

6. Oza AM, Tinker AV, Oaknin A, et al. Antitumor activity and safety of the PARP inhibitor rucaparib in patients with high-grade ovarian carcinoma and a germline or somatic BRCA1 or BRCA2 mutation: integrated analysis of data from Study 10 and ARIEL2. Gynecol Oncol. 2017;147:267-75.

7. Coleman RL, Oza AM, Lorusso D, et al. Post hoc exploratory analysis of rucaparib in patients with platinum-sensitive recurrent ovarian carcinoma from the randomized, placebo-controlled phase III study ARIEL3: effect of a deleterious germline or no germline BRCA mutation on efficacy [abstract 2402]. Presented at SGO; 16-19 March 2019; Honolulu (HI).

8. Ledermann JA, Harter P, Gourley C, et al. Olaparib maintenance therapy in patients with platinum-sensitive relapsed serous ovarian cancer (SOC) and a BRCA mutation (BRCAm). Presented at ASCO, 31 May-4 June 2013; Chicago (IL). 
9. National Institute for Health Care and Excellence. Niraparib for maintenance treatment of relapsed, platinum-sensitive ovarian, fallopian tube and peritoneal cancer: technical appraisal guidance. 2018. https://www.nice.org.uk/guidance/ta528/resources/ niraparib-for-maintenance-treatment-of-relapsed-platinumsensiti ve-ovarian-fallopian-tube-and-peritoneal-cancer-pdf-8260684719 2773. Accessed 12 Mar 2019. 